\title{
Proceso de internacionalización para la empresa Cosméticos Belier CDS SAS
}

\author{
Julián Felipe Vásquez-Rodríguez ${ }^{1}$ \\ Universidad de la Salle \\ jvasquez00@unisalle.edu.co \\ John Edison Hernández-Beltrán² \\ Universidad de la Salle \\ johnehernandez95@unisalle.edu.co \\ Jenifer Lorena Giraldo-Gómez ${ }^{3}$ \\ Universidad de la Salle \\ jennylorena289@gmail.com
}

DOI: https://doi.org/10.21158/23227230.v10.n0.2020.2944

Cómo citar este artículo: Vásquez-Rodríguez, J. F.; Hernández-Beltrán, J. E.; Giraldo-Gómez, J. L. (2020). Proceso de internacionalización para la empresa Cosméticos Belier CDS SAS. Revista Ploutos, 10, (Páginas). DOI: https://doi.org/10.21158/23227230.v10.n0.2020.2944

Fecha de recepción: 09 de septiembre de 2020

Fecha de aprobación: 25 de marzo de 2021

\section{Resumen}

Cosméticos Belier CDS SAS es una empresa colombiana, familiar y mediana dedicada a la producción y venta de productos cosméticos profesionales, enfocados en el cuidado de las manos, los pies y las uñas. Actualmente la empresa se encuentra interesada en llevar a cabo un proyecto de internacionalización debido al crecimiento constante del sector. Ante este panorama, la investigación pretende identificar un mercado potencial que contribuya al proceso de internacionalización de la empresa, analizando los riesgos y las amenazas que representa un mercado extranjero y las posibilidades de aumentar su adaptación y competitividad en un ambiente internacional. Para ello, se implementó una metodología mixta apoyada en tres fases: diagnóstico empresarial, identificación del producto estrella y selección de mercados. En estas se evaluaron variables microeconómicas y macroeconómicas de los países latinoamericanos, lo que arrojó como resultado que el país con mejor potencial para que la empresa empiece a exportar sus productos es México, ya que es un mercado que tiene una alta demanda de productos de belleza y cuenta con una baja participación de bienes cosméticos colombianos.

Palabras clave: comercio internacional; selección de mercado; diagnóstico empresarial; proceso de internacionalización; exportaciones; industria cosmética.

${ }^{1}$ Estudiante de Finanzas y Comercio Internacional - Universidad de la Salle. Integrante del Semillero de investigación Aplicado a las Finanzas y Comercio Internacional (SIAFCI). ORCID: https://orcid.org/0000-0001-8376-4097

2 Estudiante de Finanzas y Comercio Internacional - Universidad de la Salle. Integrante del Semillero de investigación Aplicado a las Finanzas y Comercio Internacional (SIAFCI). ORCID: https://orcid.org/0000-0003-2056-392X

3 Estudiante de Finanzas y Comercio Internacional - Universidad de la Salle. Integrante del Semillero de investigación Aplicado a las Finanzas y Comercio Internacional (SIAFCI). ORCID: https://orcid.org/0000-0001-7507-3368 


\title{
Internationalization process \\ for the company Cosméticos Belier CDS SAS
}

\begin{abstract}
Cosméticos Belier CDS SAS is a Colombian, family-owned, medium-sized company engaged in the production and sale of professional cosmetic products, focused on hand, foot, and nail care. Due to the constant growth of the sector, the company is currently interested in carrying out an internationalization project. Given this scenario, this research aims at identifying a potential market that contributes to the internationalization process of the company, analyzing the risks and threats posed by the foreign market and the possibilities of increasing its adaptation and competitiveness in an international environment. To this end, a mixed methodology based on three phases was implemented: business diagnosis, identification of the star product, and market selection. In these phases, microeconomic and macroeconomic variables of Latin American countries were evaluated, resulting in the conclusion that the country with the best potential for the company to start exporting is Mexico, since it is a market with high demand for beauty products and low participation of Colombian cosmetic goods.
\end{abstract}

Keywords: international trade; market selection; business diagnosis; internationalization process; exports; cosmetics industry.

\section{Processo de internacionalização \\ para a empresa Cosméticos Belier CDS SAS}

\section{Resumo}

Cosméticos Belier CDS SAS é uma empresa colombiana, familiar e de médio porte que se dedica à produção e comercialização de produtos de cosméticos profissionais, focados no cuidado das mãos, pés e unhas. Atualmente a empresa tem interesse em realizar um projeto de internacionalização devido ao constante crescimento do setor. Neste contexto, a pesquisa visa identificar um mercado potencial que contribua para o processo de internacionalização da empresa, analisando os riscos e ameaças que um mercado estrangeiro representa e as possibilidades de aumentar a sua adaptação e competitividade em um ambiente internacional. Para isso, foi implementada uma metodologia mista apoiada em três fases: diagnóstico empresarial, identificação do produto estrela e seleção do mercado. Nelas, foram avaliadas as variáveis microeconômicas e macroeconômicas dos países da América Latina, que mostraram, como resultado, que o país com maior potencial para a empresa começar a exportar seus produtos é o México, por já ter um mercado com grande demanda de produtos de beleza e baixa participação dos produtos cosméticos colombianos.

Palavras-chave: comércio internacional, seleção de mercado, diagnóstico empresarial, processo de internacionalização, exportações, indústria cosmética.

\section{Processus d'internationalisation}




\begin{abstract}
Résumé
Cosméticos Belier CDS SAS est une entreprise familiale colombienne de taille moyenne dédiée à la production et la vente de produits cosmétiques professionnels spécialisés dans le soin des mains, pieds et ongles. L'entreprise entreprend actuellement un projet d'internationalisation en raison de la croissance constante du secteur. Cet article vise à identifier un marché potentiel contribuant au processus d'internationalisation de l'entreprise au travers d'une analyse des risques et des menaces présents sur les marchés étrangers mais aussi des capacités d'adaptation et de compétitivité dans un environnement international. Une méthodologie mixte a ainsi été mise en place et séquencée en trois phases : le diagnostic commercial, l'identification du produit phare et la sélection du marché. Les variables micro et macroéconomiques des pays d'Amérique latine ont été évaluées, et le pays ayant le meilleur potentiel pour que l'entreprise y exporte ses produits est le Mexique dans la mesure où il s'agit d'un marché ayant une forte demande de produits de beauté et une faible pénétration des produits cosmétiques colombiens.
\end{abstract}

Mots-clés: commerce international ; sélection $d u$ marché; diagnostic d'entreprise; processus d'internationalisation; exportations; industrie cosmétique.

\title{
1. Introducción
}

La internacionalización de las empresas ha tomado fuerza debido a su impacto social y económico, Ortega y Espinoza (2015) afirman que esto está relacionado con la supervivencia empresarial, puesto que al estar en diferentes mercados se encuentran diversas oportunidades que no se darían de operar solo en un mercado local.

Cosméticos Belier pertenece al subsector de cosméticos, belleza y cuidado personal. Este subsector deriva del principal sector de cosméticos, aseo del hogar y absorbentes, según la Cámara de la Industria Cosmética y de Aseo de la Asociación Nacional de Empresarios de Colombia (ANDI) (2015). El sector de cosméticos en Colombia ha tenido un crecimiento regular desde el 2016. De acuerdo con los datos de Euromonitor (2018), este sector ha tenido un crecimiento del 6,5 \% en tamaño del mercado.

En tamaño por ventas es el cuarto mercado más grande de Latinoamérica —-detrás de México, Brasil y Argentina- y el principal en la región Andina en productos de cuidado personal y belleza, con ventas para el 2018 de 3422000000. 
Debido a esto, la empresa se ha interesado en llevar a cabo un proyecto de internacionalización para introducirse en el mercado latinoamericano, impulsados por diferentes motivaciones proactivas y reactivas dentro de las cuales destaca el tener un aprovechamiento de las economías a escala, de modo que esto permita a la empresa aumentar su producción y reducir sus costos, generando más competitividad.

El principal objetivo de este proyecto es orientar a la empresa con el fin de identificar un mercado potencial que contribuya al proceso de internacionalización. Para lograr este objetivo fue necesario realizar un diagnóstico empresarial que permitió valorar el nivel de preparación de la empresa con miras a empezar este proceso. Seguido de esto, se determinó qué producto del portafolio puede llegar a ser el más atractivo en un mercado internacional y, de esta manera, estar en capacidad de identificar en qué país el producto tendrá mejor acogida.

Cabe resaltar que no se encuentra información relevante sobre casos de procesos de internacionalización del sector cosméticos en Colombia, por lo que esta investigación aportará información relevante de una empresa del sector que servirá como apoyo o caso de referencia para posteriores intenciones de empresas cosméticas en Colombia dispuestas a internacionalizarse. No obstante, para esta investigación se tuvo en cuenta una gran variedad de casos empresariales de internacionalización en otros sectores que aportan, cada uno, material relevante para este estudio. A continuación se mencionaran algunos.

El caso de la empresa española Inés Rosales expone las necesidades imperantes de adaptarse al mercado destino. Al ser esta una empresa tradicional española y perteneciente a la agroindustria, se enfrentaba, principalmente, a problemas de etnocentrismo del consumidor en los mercados internacionales. Este caso, abordado y analizado por Cambra y Vázquez (2010), destaca «la habilidad de Inés Rosales para adaptarse a las características específicas de cada mercado y pone de relieve la importancia de familiarizarse con los hábitos 
de compra y de consumo de país destino y con el idioma» (p. 75). Se resalta la importancia de la adaptabilidad en un mercado dinámico y exigente.

Otro estudio desarrollado por Cornejo y Müller (2007) abarcaba de manera general las capacidades que debían tener las pymes chilenas para la inserción a mercados en Asia. A pesar de que el estudio se enfoca en el mercado asiático, se rescatan características fundamentales que mencionan allí para un proceso de internacionalización aplicables a cualquier mercado, los autores afirman que las características fundamentales que deben poseer las empresas son «capacitación de RR. HH, calidad, incorporación de tecnologías de la información y la comunicación (TIC), la capacidad para desarrollar mercados y canales de exportación, innovación, diseño de una estrategia de internacionalización y asociatividad» (p. 2).

De la mano con el anterior estudio, se reafirma la importancia que las TIC pueden llegar a representar para una pyme. Tal como lo asegura Osterlof (2011, p. 4),

las TIC son para las pymes instrumentos para acceder a una inmensa fuente de información, les permiten desarrollar procesos rápidos y confiables, y canales de comunicación inmediatos. Así como, una mayor capacidad de almacenamiento, automatización de los trabajos y más interactividad.

Es importante estimar la relevancia que han tomado las TIC en la dinámica actual del comercio internacional. La correcta utilización de estas tecnologías puede desencadenar casos de éxito. Por ejemplo, en el caso de la pyme Mr. Wonderful, una empresa española dedicada a la venta de detalles y regalos «innovadores», ha utilizado a su favor las TIC para difundir su marca y alcanzar mercados internacionales de talla europea, tales como Alemania y Francia. Esta empresa encontró en las TIC la oportunidad de diversificar sus mercados y ofrecer una experiencia más personalizada al cliente a través de su portal web.

De esta manera, se establecen los factores claves para la internacionalización de una empresa y se pretende construir a partir de ellos un plan ajustado a las necesidades y las capacidades de la empresa objeto de estudio. A fin de lograrlo se propone un método mixto 
de investigación —cuali-cuantitativa - que recoja los aspectos más importantes de ambas ramas para la construcción de una estrategia de internacionalización adaptada a la realidad y actualidad de Cosméticos Belier.

En este sentido, se encuentra que datos numéricos tales como ventas, exportaciones/importaciones de productos cosméticos y precios, entre otros, son tan importantes para esta investigación como lo son las características cualitativas de la empresa que permiten evaluar su capacidad de internacionalización.

Ahora bien, este artículo explica de forma específica la metodología utilizada en la investigación y los datos empleados a lo largo de esta. Posteriormente, se exponen los resultados obtenidos y se practica un breve análisis de estos con la finalidad de establecer la base de lo que será la estrategia de internacionalización. Por último, se concluye con las fortalezas y debilidades de la empresa con miras a encaminarse a un proceso de internacionalización.

\section{Metodología y datos}

El método de estudio que se implementó para llevar a cabo esta investigación fue descriptivo con un enfoque mixto, definido, de acuerdo con Hernández-Sampieri, MéndezValencia, Mendoza-Torres, Fernández-Collado y Baptista-Lucio (2014) como «la integración sistemática de los métodos cuantitativo y cualitativo». Estos pueden ser conjuntados de tal manera que las aproximaciones cuantitativa y cualitativa conserven sus estructuras y procedimientos originales, la «forma pura de los métodos mixtos». Alternativamente, estos métodos pueden ser adaptados, alterados o sintetizados para efectuar la investigación y lidiar con los costos del estudio, la «forma modificada de los métodos mixtos» (p. 546).

La presente investigación está dividida en tres fases. En la primera, que se denominó fase de diagnóstico, se realizó una investigación de tipo cualitativo, pues lo que se buscaba era la recolección de datos por medio de una entrevista realizada al gerente de la empresa. 
Para esto se tomó como referencia el diagnóstico de exportación que es proporcionado por Procolombia, cuyas preguntas permiten evaluar el estado de la empresa desde el ámbito financiero, administrativo, logístico y humano, para también conocer su potencial exportador y analizar sus fortalezas y las debilidades que tienen frente al mercado global. Todo esto con el propósito de estar en capacidad de analizar la capacidad que posee la empresa para ingresar en mercados internacionales y la viabilidad de que la empresa cumpla con las mínimas condiciones que le permitan realizar estos procesos de inserción.

La segunda fase es la de identificación del producto estrella. En esta se utilizó el portafolio que nos suministró Cosméticos Belier, el cual está relacionado con las ventas de sus productos para el 2019. Con base en lo anterior, se creó una matriz BCG (Boston Consulting Group), la cual es una herramienta gráfica de análisis estratégico que permite evaluar los productos del portafolio a través de criterios de crecimiento y participación. Es así como ayudó a identificar cuál es el producto estrella con el que cuenta la empresa y que puede tener mejor impacto a la hora de ingresar a un nuevo mercado.

La tercera fase consistió en la selección de mercados. Para esta se empleó una matriz de comparación que permitió evaluar diferentes aspectos microeconómicos y macroeconómicos de los países latinoamericanos preseleccionados. Estos países fueron cinco y se escogieron de acuerdo con las cifras de importación de productos cosméticos.

Entre las variables evaluadas en la matriz comparativa se analizaron factores macroeconómicos en los que se valoraron aspectos tales como el PIB, el crecimiento del PIB, el PIB per cápita, la inflación y la población activa femenina mayor a 15 años. En los factores microeconómicos se apreciaron aspectos relacionados con el sector cosmético como, por ejemplo, importaciones del producto desde Colombia, el número de industrias, el arancel, la logística y el crecimiento del producto, analizados gracias a los datos obtenidos del Banco Mundial y Trade Map. 


\section{Resultados}

\subsection{Diagnóstico de exportación}

De acuerdo con las preguntas realizadas en el diagnóstico de exportador al gerente de la compañía se practicó el siguiente análisis de los datos recabados. Los siguientes gráficos recogen las respuestas a las preguntas realizadas. Se asume que entre mayores respuestas positivas — sí- tenga cada sección, mayor será la favorabilidad de este.

Figura 1. Condiciones de la compañía

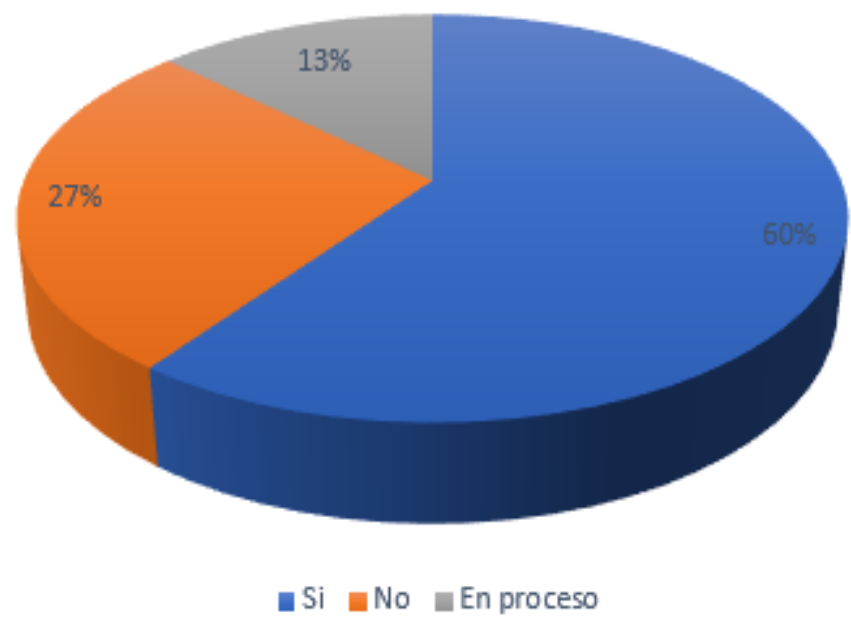

Fuente. Elaboración propia.

En la figura 1 se evaluaron las condiciones generales de la compañía en aspectos tales como la productividad, la experiencia, la competitividad y los buenos estándares de calidad.

De acuerdo con los resultados obtenidos, se evidenció que cuentan con información financiera útil para la toma de decisiones, que puede servir para definir capacidad de inversión y costo del producto a exportar. Sin embargo, se sugiere actualizar los datos y crear unas cuentas contables de costos acordes con el presente de la compañía, ya que se encuentran algunas deficiencias en la contabilización de algunos productos. Por lo pronto, 
los estados financieros de la compañía funcionan como herramienta para la toma de decisiones.

En el aspecto productivo existe claridad en el producto a exportar y su capacidad productiva. Cuentan con un laboratorio cosmético propio, el cual les garantiza una capacidad de producción del $50 \%$ destinada a productos exportables, equivalente a 45000 unidades de esmalte mensuales. No obstante, hace falta definir con exactitud procesos productivos eficientes y una estructura de costos sólida a fin de elaborar una oferta productiva y obtener una herramienta financiera para la toma de decisiones.

Tienen como debilidad su cadena de abastecimiento, puesto que manifiestan indicios de inestabilidad y poca sinergia con algunos proveedores a fin de garantizar insumos. Aseguran que se encuentran trabajando en estrategias para entablar relaciones comerciales sólidas con proveedores nacionales, de modo que puedan conseguir mejores condiciones de pago y abastecimiento oportuno.

Figura 2. Herramientas comerciales

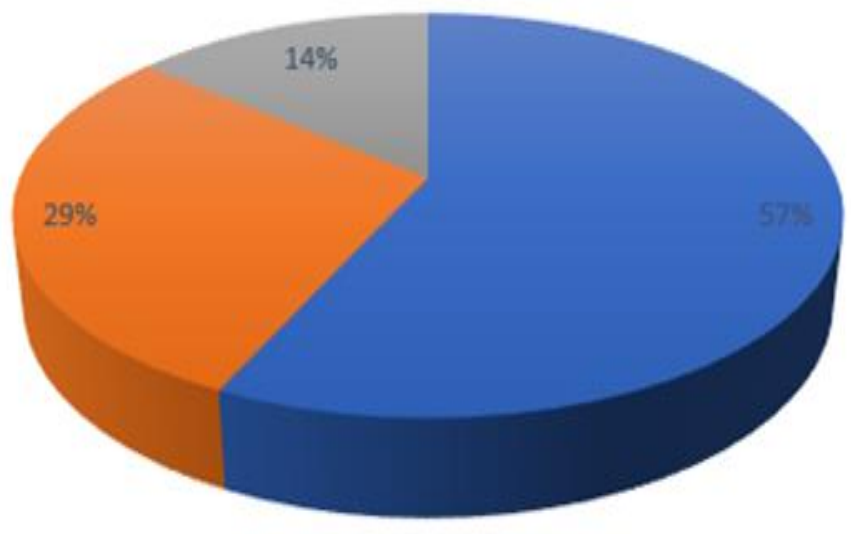

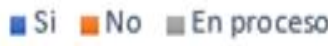

Fuente. Elaboración propia. 
En esta sección se encuentra un panorama positivo, dado que la empresa cuenta con un departamento exclusivo de marketing liderado por un gerente de área con dominio de lengua extranjera. En este departamento administran el funcionamiento de la página web que cuenta con opción de compras online. Esta herramienta será de vital importancia, dadas las tendencias actuales de consumo, así como servirá de apoyo para la visibilidad de la marca en mercados internacionales.

Desde el aspecto comercial se encuentran en proceso de adaptación a mercados nacionales, lo que reflejan que esta empresa tendría una buena disposición para adaptación a nuevos mercados. Sin embargo, el personal no cuenta con dominio de lengua extranjera o con experiencia en ventas internacionales, lo que representa una debilidad en esta área.

Figura 3. Experiencia exportadora

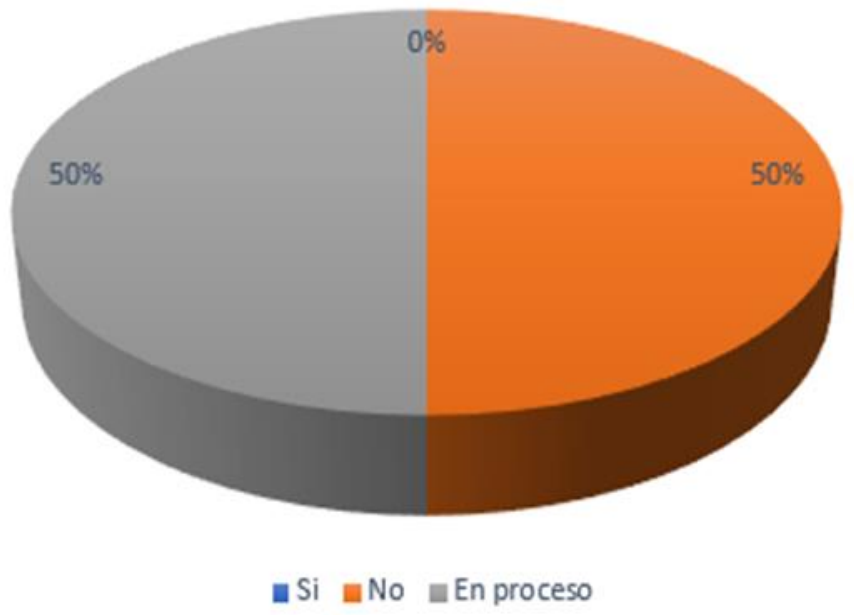

Fuente. Elaboración propia.

Los resultados obtenidos muestran que esta empresa no ha realizado ninguna exportación directa, pero se encuentran en proceso de envíos de muestras sin valor comercial a mercados internacionales en los que han empezado a ofrecer el portafolio de la compañía. Manifiestan haber participado en ferias internacionales organizadas por 
entidades del estado como Procolombia y Colombia Productiva, así como aseguran tener una base de datos de clientes potenciales en el exterior, lo que puede facilitar la inserción al mercado objetivo.

Dado que la empresa no cuenta con experiencia exportadora se busca que su primera operación tenga éxito a través de un producto estrella que garantice condiciones favorables para convertir una primera exportación en la base para relaciones comerciales sólidas con otros mercados y expandir la difusión de la marca.

\subsection{Identificación del producto estrella}

Basada en el portafolio de ventas mensuales del 2019 de Cosméticos Belier se generó la tabla 1.

Tabla 1. Cantidad productos portafolio

\begin{tabular}{|c|c|}
\hline Grupo & Cantidad de datos \\
\hline Accesorios para cuidado & 55 \\
\hline Esmaltes & 266 \\
\hline Artículos de aseo & 19 \\
\hline Artículos para decoración & 29 \\
\hline Utensilios para uñas & 43 \\
\hline Maletines y carteras & 6 \\
\hline Otros & 5 \\
\hline Total & 423 \\
\hline
\end{tabular}

Fuente. Elaboración propia.

La tabla 1 corresponde al portafolio de productos que fueron ofrecidos por la empresa, el cual se divide en siete grupos distintos que abarcan 423 productos en total. A continuación, se muestran los productos que obtuvieron una mayor participación de ventas registradas de la empresa para el 2019. 
Tabla 2. Ventas de productos de Cosméticos Belier

\begin{tabular}{|c|c|}
\hline Producto & Unidades vendidas \\
\hline Esmaltes & $\mathbf{2 0 1 9}$ \\
\hline Accesorios para el cuidado & 17110 \\
\hline Artículos para decoración & 7779 \\
\hline Utensilios uñas & 7511 \\
\hline
\end{tabular}

Fuente. Elaboración propia.

A través de la matriz BCG se busca definir el producto estrella, el producto interrogante, el producto vaca y el producto perro.

Figura 4. Matriz BCG

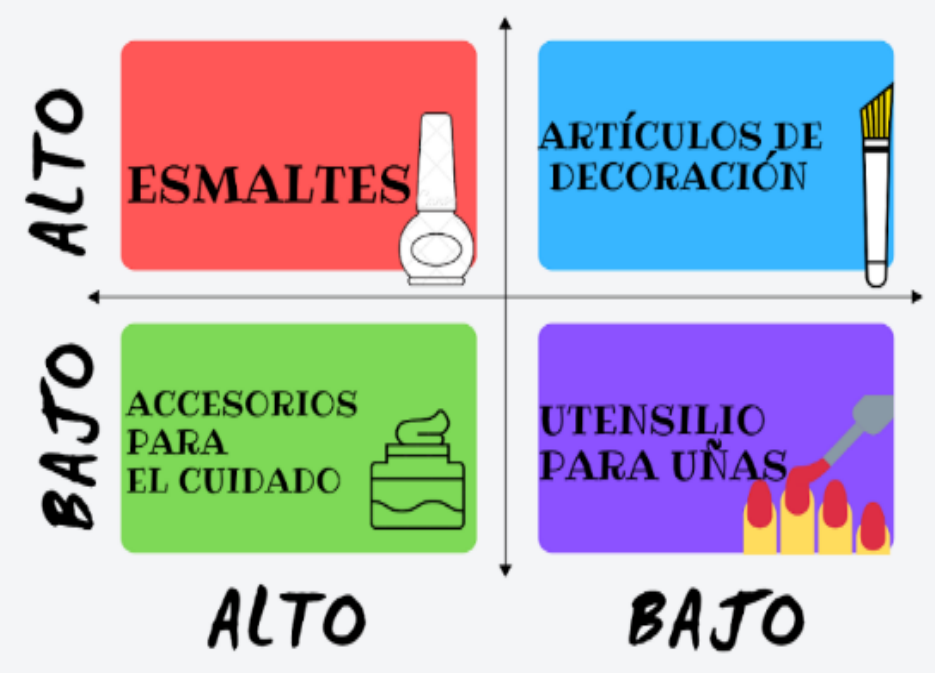

Fuente. Elaboración propia.

Según la matriz, los esmaltes se ubican como el producto estrella para incursionar en el mercado objetivo. porque conservan un mayor potencial de crecimiento y sus ventas aumentan en los últimos años -2016-2019-. De igual manera, se considera un producto dinámico de alta rotación con picos de ventas estacionales en meses como marzo, junio, septiembre y diciembre. 
El producto interrogante se asigna a los artículos de decoración, dado que su crecimiento reciente en ventas se debe al impulso que ha implementado la compañía en los cursos de manicura que dictan ocasionalmente en sus redes sociales y en conjunto con academias de belleza. Por ende, su participación en ventas no es significativa, sin embargo, se mantiene como proyecto de maduración de marca y servicio.

El producto vaca o producto con alta cuota de mercado y baja tasa de crecimiento corresponde a los accesorios para el cuidado. Aquí se encuentran las cremas humectantes y los aceites humectantes, entre otros productos complementarios para la manicura. Se consideran productos ya madurados en el mercado nacional y perfilados para una inserción al mercado internacional cuando se haya alcanzado una cuota de mercado en el mercado objetivo.

Por último, el producto perro o aquel que tiene baja cuota de mercado y crecimiento son los utensilios para uñas. Aquí encontramos utensilios tales como limas, cortaúñas, cortacutículas, etc., que se consideran complementarios y se ofrecen como kits a un cliente profesional. Por tanto, no existe intención pronta por ingresar estos productos al mercado internacional.

\subsection{Selección de mercado objetivo}

Ahora bien, para este caso se considera mercado objetivo aquel que tenga condiciones similares al nacional y cumpla con cifras microeconómicas y macroeconómicas favorables para exportación desde Colombia del producto en mención. Es por esto que en esta preselección solo se tienen en cuenta países de la región. En primer lugar, se identifica qué país latinoamericano importa más productos con la subpartida arancelaria 33.04.30. Aquellos cinco países con mayores importaciones se filtraron para generar la matriz comparativa y así definir el mercado objetivo. Las importaciones del producto nos permitirán evaluar qué países tienen un mayor consumo del bien que la empresa desea exportar. Según 
la base de datos Trade Map los países con mayor demanda del producto son los que se presentan en la tabla 3 .

Tabla 3. Importaciones mundiales de la subpartida 33.04 .30 preparaciones para manicuras o pedicuros

\begin{tabular}{|c|c|}
\hline Importadores & $\begin{array}{c}\text { Valor importado 2019 } \\
\text {-miles de dólares- }\end{array}$ \\
\hline México & 27524 \\
\hline Chile & 17903 \\
\hline Perú & 7813 \\
\hline Ecuador & 6236 \\
\hline Costa Rica & 4815 \\
\hline
\end{tabular}

Fuente. Elaboración propia con datos Trade Map.

Las exportaciones realizadas desde Colombia hacia los cinco países más importadores servirán para encontrar qué tanta participación del producto colombiano existe y qué país ofrece una mejor oportunidad.

Tabla 4. Exportaciones desde Colombia a los países más importadores de la subpartida 33.04.30

\begin{tabular}{|c|c|}
\hline Exportadores & $\begin{array}{c}\text { Valor exportado 2019 } \\
\text {-miles de dólares- }\end{array}$ \\
\hline Ecuador & 3917 \\
\hline Perú & 2875 \\
\hline Chile & 1891 \\
\hline México & 909 \\
\hline Costa Rica & 895 \\
\hline
\end{tabular}

Fuente. Elaboración propia con datos de Trade Map.

De acuerdo con las tablas 3 y 4, se encuentra que México es el mayor importador de la región de esta subpartida con 29650 miles de dólares importados en el 2019, de los cuales 909 miles de dólares importados son provenientes de Colombia. Ecuador, por su parte, se posiciona como el principal consumidor de estos productos provenientes de Colombia. 
Ambos mercados, tanto el mexicano como el ecuatoriano, se consideran con similitudes amplias en cuanto a cultura de consumo y aceptación de productos.

Los anteriores países son sometidos a un análisis de variables macroeconómicas y microeconómicas con el fin de identificar mayores oportunidades de exportación.

Tabla 5. Matriz comparativa

\begin{tabular}{|c|c|c|c|c|c|c|c|c|}
\hline \multicolumn{3}{|c|}{ PONDERACIÓN } & VARIABLES & MEXICO & CHILE & PERÚ & ECUADOR & $\begin{array}{l}\text { COSTA } \\
\text { RICA }\end{array}$ \\
\hline \multirow{5}{*}{$65 \%$} & \multirow{5}{*}{ Microeconómica } & $25 \%$ & Importaciones desde Colombia & 2 & 3 & 4 & 5 & 1 \\
\hline & & $10 \%$ & $\begin{array}{l}\text { Número de industrias del } \\
\text { sector cosméticos }\end{array}$ & 4 & 3 & 5 & 2 & 1 \\
\hline & & $16 \%$ & Arancel a Colombia & 5 & 4 & 3 & 2 & 1 \\
\hline & & $19 \%$ & Logística & 5 & 4 & 3 & 2 & 1 \\
\hline & & $30 \%$ & $\begin{array}{l}\text { Crecimiento de importaciones } \\
\text { del producto }\end{array}$ & 4 & 5 & 2 & 3 & 1 \\
\hline \multirow{5}{*}{$35 \%$} & \multirow{5}{*}{ Macroeconómica } & $18 \%$ & PIB & 5 & 4 & 3 & 2 & 1 \\
\hline & & $20 \%$ & Crecimiento del PIB & 1 & 3 & 5 & 2 & 4 \\
\hline & & $20 \%$ & PIB per cápita & 5 & 3 & 4 & 1 & 2 \\
\hline & & $15 \%$ & $\begin{array}{l}\text { Población activa femenina } \\
\text { mayores } 15 \text { años }\end{array}$ & 2 & 3 & 5 & 4 & 1 \\
\hline & & $27 \%$ & Inflación & 5 & 4 & 3 & 1 & 2 \\
\hline \multicolumn{4}{|c|}{ PUNTAJE } & 4,004 & 3,771 & 3,461 & 2,499 & 1,266 \\
\hline
\end{tabular}

Fuente. Elaboración propia con datos del Banco Mundial y Trade Map.

De acuerdo con la tabla 5 de comparación, se obtuvieron los puntajes que se presentan en la tabla 6.

Tabla 6. Resultados por país matriz de comparación

\begin{tabular}{|c|c|}
\hline País & Calificación \\
\hline México & 4,004 \\
\hline Chile & 3,771 \\
\hline Perú & 3,461 \\
\hline Ecuador & 2,499 \\
\hline Costa Rica & 1,266 \\
\hline
\end{tabular}

Fuente. Elaboración propia. 
Como se puede observar en la tabla 7, el país con mayor puntuación es México, por lo cual es conveniente determinar que México es el país con mayor potencial para exportar los productos de la empresa Cosmético Belier, debido a que es un mercado que no tiene una alta participación de productos cosméticos colombianos y es una oportunidad de diversificar los productos ofrecidos por este mercado. Además, dada la distancia psicológica que se tiene con México, se presta para consolidarse en un segmento de mercado similar al del mercado local.

\section{Conclusiones}

En un proceso de internacionalización en el que se evalúan aspectos tanto a nivel interno de la compañía como externo, Cosméticos Belier se muestra sólido en el ámbito interno, desde su capacidad productiva hasta la experiencia del recurso humano, especialmente en el departamento de marketing.

Sin embargo, la empresa presenta debilidades en otros ámbitos cruciales, como, por ejemplo, en su cadena de abastecimiento y eficiencia productiva. Es necesario trabajar en el fortalecimiento de las relaciones con proveedores, mejorar la cadena productiva y hacer un estudio de costos y presupuesto de producción, a fin de lograr mayor eficiencia de cara a una eventual internacionalización. Esto permitirá tener información de primera mano para la toma de decisiones en aspectos productivos y comerciales.

Adicional a lo anterior, Cosméticos Belier debe invertir tiempo en el mejoramiento de sus procesos internos. Es recomendable empezar a aplicar un sistema de gestión en el que se apliquen documentos y formatos formales para cada proceso de la empresa. Así se logrará mayor eficiencia en procesos internos y mayor competitividad en el propósito de ingresar a un mercado internacional.

El producto estrella de la empresa se presta para adaptarse a las dinámicas y tendencias de consumo, de modo que el departamento de investigación y desarrollo debe estar a la vanguardia de las nuevas tendencias y programar lanzamientos periódicos que 
garanticen un impacto de la marca en el mercado objetivo. Con esto se envía un mensaje de dinamismo de la empresa frente a las condiciones de mercado y adaptabilidad en este.

Por último, el mercado objetivo seleccionado se perfila como una opción atractiva para las condiciones actuales de Cosméticos Belier. Se destaca la distancia psicológica cercana con este mercado, en el que las tendencias de consumo son similares al mercado doméstico. El reto para la empresa será potencializar las bondades y características de su producto a fin de generar un impacto en México y aspirar a un crecimiento sostenido en ventas.

\section{Referencias}

Cámara de la Industria Cosmética y de Aseo (2015). Informe de Sostenibilidad 2015 Industria Cosmética y de Aseo. Colombia. Recuperado de https://bit.ly/3haLeFn

Cambra, J.; Vázquez, R. (2010). Inés Rosales el reto de internacionalizar la actividad de una pyme es posible. Universia Business Review, (28), 62-77.

Cornejo, D.; Müller, T. (2007). Capacidades para la internacionalización asiática de las pymes chilenas (Tesis de grado). Universidad de Chile. Santiago de Chile, Chile. Recuperado de https://bit.ly/2TqceYx

Euromonitor (2018). Colour Cosmetics in Colombia, country report.

Hernández-Sampieri, R.; Méndez-Valencia, S.; Mendoza-Torres, C. P.; Fernández-Collado, C.; BaptistaLucio, M. del P. (2014). Metodología de la investigación. México: McGraw Hill.

Ortega, A.; Espinoza, J. (2015). Plan de internacionalización empresarial. España: Esic Editorial.

Osterlof, D. (2011). Las TIC como instrumento para acceder al mercado mundial. Red Latinoamericana de Política Comercial - SerieBrief, (78), 1-6. 